\title{
Controlling Iron Deficiency Anemia Through The Use of Home-fortified Complementary Foods
}

\author{
Stanley H. Zlotkin, Anna L. Christofides, S.M. Ziauddin Hyder, Claudia S. Schauer, \\ Mélody C. Tondeur and Waseem Sharieff \\ Departments of Pediatrics, Nutritional Sciences and Health Policy, Management and Evaluation and Centre for \\ International Health, University of Toronto, Division of Gastroenterology and Nutrition, Programs in Metabolism \\ and Integrative Biology, Research Institute, The Hospital for Sick Children, Toronto, Canada
}

\begin{abstract}
Iron deficiency anemia (IDA) is more common in South Asian countries including India, Bangladesh and Pakistan than anywhere else in the world. During infancy and early childhood, IDA is associated with impaired psycho-motor development and cognitive function that may be irreversible. As a consequence, there is a growing awareness that IDA is one of many factors impeding socio-economic prosperity of developing nations. The combination of unacceptably high prevalence rates and inadequate preventative programs highlights the need for new effective sustainable strategies to control IDA. The burden of iron deficiency can be reduced by taking a more holistic approach that would include promotion of healthy weaning practices and use of appropriate complementary foods, together with improving the nutritional value of such foods. There is an increasing body of peer-reviewed literature to support the contention that "micronutrient Sprinkles" is an effective strategy to improve the nutritional value of home-prepared complementary foods and thus to reduce the burden of iron deficiency among children. By combining data from recently conducted randomised control trials, Sprinkles were shown to be as efficacious as iron drops for treating childhood anemia. The iron in Sprinkles is well absorbed, and Sprinkles are easy to use and well accepted by young children and their caregivers. Integrated into existing public health programs, Sprinkles has the potential to improve the effectiveness of such programs. [Indian J Pediatr 2004; 71 (11) : 1015-1019] E-mail: Stanley.Zlotkin@sickkids.ca
\end{abstract}

Key words : Complementary feeding; Sprinkles; Iron

The burden of Iron Deficiency Anemia in Young Children in South Asia

Iron deficiency is the most common preventable nutritional deficiency in the world, especially among infants and young children in developing countries. ${ }^{1}$ Its associated anemia is linked with depressed mental and motor development during infancy and early childhood, which may be irreversible. ${ }^{2}$ Iron deficiency anemia (IDA) during childhood also results in decreased physical activity and decreased interaction with the environment, with negative consequences on learning and school achievements. ${ }^{3}$ Prevalence of IDA among children under 5 years of age in South Asia is estimated to be 75\%, 55\% and $56 \%$ in India, Bangladesh and Pakistan, respectively. ${ }^{4}$, ${ }^{5}$ It is noteworthy that these prevalence estimates (and estimates found in other reports) were based on hemoglobin concentrations alone and that iron deficiency was assumed to be the main cause of anemia. These prevalence rates are unacceptably high compared, for example, to $<5 \%$ in Canada. ${ }^{6}$ Furthermore, the burden of IDA is likely to be even higher among younger children.

Correspondence and Reprint requests : Dr. Stanley H. Zlotkin, Division of Gastroenterology and Nutrition, The Hospital for Sick Children, 555 University Avenue, Toronto, ON M5G 1X8, Canada. Fax : 4168134972.

Indian Journal of Pediatrics, Volume 71-November, 2004
For example, the 1998-99 National Family Health Survey2 (NFHS-2) in India documented a prevalence of IDA of $75 \%$ among children 6-35 months of age. ${ }^{7}$ Similarly, a recent survey in Bangladesh found that $84 \%$ of the children aged 6-24 months had IOA. ${ }^{8}$

The cause of iron deficiency among young children is multi-factorial, however the consumption of foods with low bio-available iron is likely the most important factor, especially in developing countries. ${ }^{9}$ Iron intake from complementary foods is critical since as much as $98 \%$ of the iron must be provided from these foods. ${ }^{9}$ The recent WHO/UNICEF review of complementary foods in developing countries concluded that iron requirements may be difficult to meet from non-fortified complementary foods, especially if animal products are not widely consumed..$^{10}$ In addition, in South Asia, prolonged exclusive breastfeeding, delayed introduction of complementary foods (which are often of poor quality) and feeding inappropriately small amounts of these foods to infants likely contribute to iron deficiency. ${ }^{9,11,12}$ These inadequate weaning practices may be a result of a combination of factors including lack of education, unfounded cultural beliefs and most importantly inadequate access to nutrient-dense foods.

In recent years, there has been a growing awareness that IDA not only impairs health but through its effects on 


\section{Stanley H. Zlotkin et al}

cognition and thus educational achievement among young children, translates into decreased adult productivity and ultimately economic prosperity. ${ }^{13} \mathrm{In}$ economic terms, Horton and Ross estimated the magnitude of loss of work productivity due to childhood anemia to be $4.5 \%$ of the country's Gross Domestic Product (GDP)..$^{13}$ The latest estimates of the percentage of GDP lost to all forms of vitamin and mineral deficiencies including IDA are 1\% for India, $0.9 \%$ for Bangladesh and $1.7 \%$ for Pakistan. ${ }^{4}$ Thus, clearly, there is an urgent need to develop and implement effective strategies to reduce the burden of iron deficiency through public health programs.

\section{EXISTING PUBLIC HEALTH PROGRAMS FOR CONTROLLING IRON DEFICIENCY ANEMIA IN YOUNG CHILDREN}

Unfortunately, it is apparent from the high prevalence estimates that current strategies implemented in South Asia have not proven to be effective in reducing the burden of childhood anemia. The reasons appear manifold. Even though iron deficiency anemia has been recognized as a major public health problem among children in India, low priority has been given for its prevention and control. ${ }^{5}$ In 1998-99, for example, the National Anemia Control Program reached fewer than $28 \%$ of Indian children 1-5 years of age with small ironfolic acid tablets. ${ }^{5}$ Moreover, these pediatric tablets were cumbersome to administer to the younger children who had difficulty in swallowing them. ${ }^{14}$ Thus, the combination of low coverage and poor adherence to the intervention is likely to be the primary reason why the prevalence of IDA remains high among young children in India even after the implementation of the National Anemia Control Program. As an alternative to the pediatric tablets, iron drops are currently recommended for younger children in India (and elsewhere in the world).$^{14}$ However, existing evidence suggests that iron drops are unlikely to be effective in the control of childhood iron deficiency anemia partly due to poor compliance and ineffective distribution. ${ }^{15-17}$ Indeed, adherence to the use of iron drops is impacted by the gastrointestinal side effects, unpleasant and strong metallic taste, staining of a child's teeth if not wiped off immediately and complicated dispensing instructions. ${ }^{3}$ There are also technical disadvantages associated with the use of liquid iron preparations such as limited shelf life and expensive transportation costs due to the weight of the bottles. ${ }^{15}$

Other interventions in South Asia are centred around a food-based strategy which include promotion of better weaning practices including the timely introduction and adequate feeding of complementary foods in addition to breast milk; dietary diversification to increase the availability, access and utilization of foods with a high quantity and high bioavailability of micronutrients. The prevention of childhood iron deficiency and anemia based on a food-based strategy is critical since it is the only sustainable method of preventing malnutrition. ${ }^{18}$ Such approaches are currently encouraged in India. For example, the promotion of iron-fortified foods (like fortified cereals) is recommended where it is feasible, however no cost-effective technologies are currently available, ${ }^{14}$ this means that only a minority of families in India can purchase commercially prepared fortified foods. Programs promoting healthy weaning practices including the timely introduction of complementary foods in addition to breast milk through intense nutrition and health education are currently underway in India. ${ }^{19}$ In fact, by 2007, the National Nutrition Goals for the Tenth Five-Year Plan include increasing the complementary feeding rate, at 6 months of age, from the current level of $33.5 \%$ to $75 \%{ }^{19}$ However, as previously noted, complementary foods are poor sources of dietary iron in most developing countries including India. ${ }^{9}$ According to Gibson et al, ${ }^{9}$ even if strategies to improve iron intake and bioavailability are used in plant-based complementary foods, they may not be sufficient to overcome the deficits in iron.

Therefore, in 2002, a technical consultation was organized by the Human Nutrition Unit, All India Institute of Medical Sciences, New Delhi to review existing iron interventions and set goals to control childhood anemia in India. ${ }^{14}$ Participants at that meeting reaffirmed the importance of providing young children with adequate amounts of iron and suggested areas for future research, including the fortification of complementary foods.

\section{SPRINKLES: A NEW APPROACH TO IMPROVE THE MICRONUTRIENT CONTENT OF HOME-PREPARED COMPLEMENTARY FOODS}

Controlling iron deficiency is consistent with the aim of our research group at the Hospital for Sick Children, University of Toronto. We believe that the burden of IDA can be reduced by taking a more holistic approach that would include promotion of healthy weaning practices and use of appropriate complementary foods, together with improving the nutritional value of such foods. Thus, we strongly support the WHO recommendation of exclusive breastfeeding followed by the introduction of nutrient-rich complementary foods at six months of age with the continuation of breastfeeding up to two years of age and beyond. ${ }^{20}$ To improve the nutritional value of home-prepared complementary foods, "micronutrient Sprinkles" in a powder form were developed as a homefortification strategy for improving the nutritional quality of home-prepared complementary foods. They are formulated to contain microencapsulated ferrous fumarate along with other micronutrients according to the needs of the target population including zinc, vitamins $A, C$ and D or folic acid. To administer the 


\section{Controlling Iron Deficiency Anemia Through the Use of Home-Fortified Complementary Foods}

correct amounts of iron and other micronutrients, Sprinkles are packaged in single-dose-sachets. Caregivers are instructed to add the entire contents of one sachet daily onto any semi-solid food served to their infants in the household. The iron in Sprinkles is encapsulated with a thin coating of a vegetable-based hydrogenated lipid that prevents the iron from oxidizing the food so as not to change the color, texture or taste of the food to which Sprinkles are added.

To evaluate the efficacy, acceptability and safety of Sprinkles in diverse settings and to determine the amount of iron absorbed from Sprinkles, we conducted several community based trials using Sprinkles in five different countries involving over 2,000 anemic, iron deficient and iron sufficient infants and young children. Clinical trials in a rural setting are currently ongoing in partnership with the King Edward Memorial Hospital and Medical College in Pune, India. These studies aim to further investigate the optimal iron dose and duration of the Sprinkles intervention.

\section{(i) Efficacy and Bioavailability}

The first randomized controlled trial (RCT) was carried out in a community setting in rural Ghana (a malaria endemic area) where we compared Sprinkles to iron drops given daily (current treatment standard in a clinical setting) among 557 infants 6-18 months of age with hemoglobin in the range of 70-99 g/L. ${ }^{21}$ Approximately $60 \%$ of infants raised their hemoglobin concentration to above $100 \mathrm{~g} / \mathrm{L}$ in both groups after 2 months of intervention. To evaluate whether a 2- month intervention was long enough to prevent recurrence of anemia in infants and young children at risk, these $60 \%$ children (820 months of age) who responded to the initial intervention were randomly assigned to Sprinkles, iron drops or placebo daily for another 6 months. ${ }^{22}$ The nonanemic children at the end of this second intervention were re-assessed 12 months later. Results suggested that, in most children successfully treated for anemia with a 2month intervention, further supplementation was not needed to maintain their non-anemic status.

In another similar RCT conducted in rural Ghana during the dry season when malaria rates are low, we administered different formulations of Sprinkles (with or without zinc) to 304 infants 6-18 months of age who had hemoglobin in the range of 70-99 $\mathrm{g} / \mathrm{L} .{ }^{23}$ At the end of the 2-month intervention, approximately $75 \%$ of infants given Sprinkles without zinc had hemoglobin $\geq 100 \mathrm{~g} / \mathrm{L}$ and $63 \%$ of infants given Sprinkles with zinc had hemoglobin $\geq 100 \mathrm{~g} / \mathrm{L}$. Elsewhere, in non-malaria endemic areas, we observed that: (i) $91 \%$ of the 62 children aged 1-6 years who had anemia raised their hemoglobin to $>125 \mathrm{~g} / \mathrm{L}$ after receiving Sprinkles for 2 months in Bolivia (unpublished); and (ii) in Bangladesh, among 70 anemic young children 12-24 months old (with very little hookworm infestation) given Sprinkles for 2 months, mean hemoglobin increased from $97 \mathrm{~g} / \mathrm{L}$ to $113 \mathrm{~g} / \mathrm{L}^{24}$
To determine the optimal amount of iron to include in the Sprinkles sachet, the bioavailability of the microencapsulated iron was determined using intrinsically labelled iron and a dual-stable isotope method. ${ }^{25}$ Infants were classified as anemic, iron deficient or iron sufficient based on hemoglobin and soluble transferrin receptor concentrations. We demonstrated that the microencapsulated iron in Sprinkles was bioavailable and that infants with IDA absorbed iron from Sprinkles about twice as efficiently as those who were iron deficient without anemia or those who were iron sufficient. Based on these data, computer simulations and dose-response studies were carried out to determine the most appropriate dose of iron to include in a Sprinkles sachet and the most appropriate duration of intervention. ${ }^{26}$ It was estimated that the consumption of 60 Sprinkles sachets (each containing $12.5 \mathrm{mg}$ of elemental iron) over at least 2 months should be adequate for repletion and maintenance of sufficient iron stores and hemoglobin levels among young children.

We combined data from two of our RCTs that compared Sprinkles with iron drops given over 2 months to further evaluate the use of Sprinkles. ${ }^{21,26}$ A total of 836 infants with hemoglobin $<100 \mathrm{~g} / \mathrm{L}$ were included in the pooled analysis which concluded that Sprinkles were as efficacious as iron drops under controlled settings. ${ }^{27}$ Hemoglobin distribution curves before and after different Sprinkles interventions clearly indicated that the hemoglobin response was similar to that of iron drops (Fig 1).

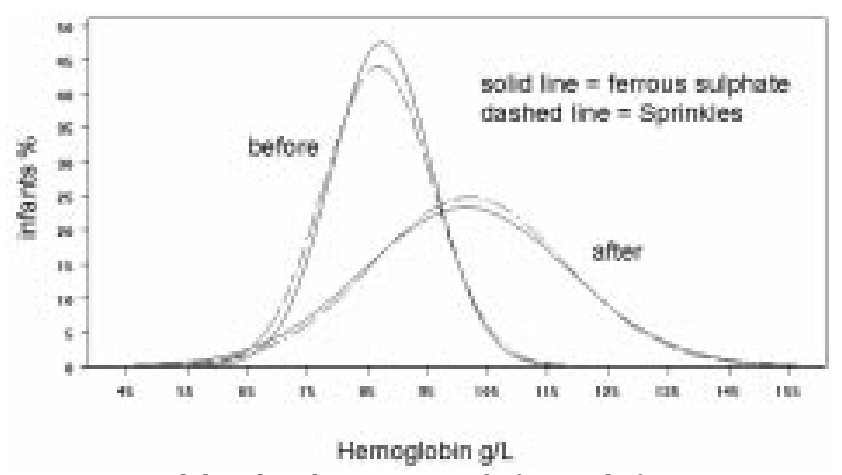

Fig. 1. Hemoglobin distribution curves before and after intervention

\section{(ii) Acceptability, Compliance and Safety}

Alongside efficacy data, information regarding usage and side effects was collected in all of our trials. Almost all caregivers reported that Sprinkles were easy to use, that there were no appreciable changes in the taste or smell of the infants' food to which Sprinkles were added, and that the sachets were more acceptable than the iron drops. ${ }^{21-24,}$ 26,28 In a few trials, a relatively high percentage of caregivers reported that Sprinkles changed the colour of their infant's food (much like the effect of adding condiment such as pepper to food). ${ }^{22-24,26}$ However, these changes did not prevent the child from eating the food to 
which Sprinkles were added. No staining of teeth was reported; side effects such as darkening of the infant's stool, constipation and diarrhea were occasionally reported in all trials. The average number of Sprinkles sachets consumed per child out of the total assigned during our trials was about $70 \%$ (range $50 \%-100 \%$ ). These data suggest that Sprinkles were well accepted in the communities that received the intervention.

We also studied the potential negative effects of iron provided as Sprinkles on growth and the risk of developing iron overload. In a multi-centre RCT conducted in Northern Canada, we provided Sprinkles or placebo for 6 months to young children aged 4-18 months who had hemoglobin concentrations $\geq 100 \mathrm{~g} / \mathrm{L} .{ }^{28}$ We did not observe significant differences in weight or height for age, or weight for height z-scores compared to similar children who received placebo. No child had any evidence of iron overload (serum ferritin concentration > $300 \mu \mathrm{g} / \mathrm{L}$ ). In a school setting in Northern China, we studied healthy children aged 3-6 years who were given Sprinkles either weekly or daily for one school term (13 weeks) or not given any Sprinkles during meals served at school. ${ }^{29}$ Results suggested that children were not at risk for developing iron overload even though their iron stores were sufficient at the beginning of the school term. Thus, based on these findings, we have concluded that Sprinkles are safe and simple to use, generally acceptable to most caregivers and their infants, and can be provided to children at home or school.

\section{IMPLEMENTING A COMPREHENSIVE APPROACH TO CONTROL IRON DEFICIENCY ANEMIA IN YOUNG CHILDREN}

Integrating alternative, innovative approaches within complementary feeding programs to lower the high burden of iron deficiency among young children is, we believe, one way to rapidly tackle this major public health problem. "Micronutrient Sprinkles" could be integrated into such complementary feeding programs to enhance the overall nutritional quality of these weaning foods. Indeed, the mixing of Sprinkles into complementary foods can enrich the foods not only with iron but also with other essential micronutrients. The sachets are simple to distribute and use, and could be incorporated as an adjunct to any program aiming to improve weaning practices in South Asia. Preventing IDA in young children will either directly or indirectly contribute towards achieving at least four out of the eight Millennium Development Goals, including: i) eradicating extreme poverty and hunger through improved productivity in adulthood; (ii) achieving universal primary education though increased learning during childhood; (iii) promoting gender equality and empowering women though gaining knowledge about proper weaning practices; and finally (iv) reducing child mortality by preventing death due to severe anemia.

\section{CONCLUSION}

There is an urgent need to promote healthy weaning practices and the consumption of nutritionally sound, low-cost complementary foods to prevent the development of iron deficiency among infants and young children in developing countries including South Asia. Sprinkles can be integrated into currently functioning complementary feeding programs promoting child health and good nutrition to enhance the nutritional quality of complementary foods directly in the home or at school, with simple instructions and at a low cost. In addition, other essential micronutrients can be provided through Sprinkles according to the specific needs of the target population. The promotion of healthy weaning practices including the use of Sprinkles has the potential to significantly decrease the prevalence of iron deficiency and IDA among young children, which will help to achieve at least four out of the eight Millennium Development Goals.

\section{Acknowledgement}

The research describing the development of Sprinkles was generously supported by grants from USAID's OMNI Research Program through the Human Nutrition Institute of the ILSI Research Foundation, the Canadian Institutes of Health Research (CIHR), Health Canada, the International Atomic Energy Agency (IAEA), and the HJ Heinz Company Foundation.

\section{Conflict of interest}

Dr. Zlotkin is an occasional consultant to Bristol Myers, Squibb, Mead Johnson Ltd. and General Foods in Canada. Dr. Zlotkin owns the intellectual property rights to Sprinkles. The HJ Heinz Company Ltd. is supporting the technical development of the Sprinkles on a cost- recovery basis.

\section{REFERENCES}

1. UNICEF. Delivering essential micronutrients: Iron. Available at: http://www.unicef.org/nutrition/index_iron.html (Accessed on August 1, 2004)

2. Grantham-McGregor S, Ani C. A review of studies on the effect of iron deficiency on cognitive development in children. J Nutr 2001; 131 : 649S-666S; discussion 666S-668S.

3. Schauer C, Zlotkin S. Home fortification with micronutrient sprinkles-a new approach for the prevention and treatment of nutritional anemias. Pediatr Chil Health 2003; 8 : 87-90.

4. MI/UNICEF. Vitamin and mineral deficiency. A global progress report. 2004

5. Proceedings from the national workshop on methodologies for assessment of vitamin A deficiency, iron deficiency anemia and iodine deficiency disorders, New Delhi, September 13-15, 2004. All India Institute of Medical Sciences, India, 2004.

6. Zlotkin SH, Ste-Marie M, Kopelman H, Jones A, Adam J. The prevalence of iron depletion and iron-deficiency anaemia in a randomly selected group of infants from four Canadian cities. Nutr Research 1996; 16 : 729-733.

7. National Family Health Survey-2 (NFHS-2)- India 1998-99. Key findings: Anemia among women and children. Mumbai, International Institute for Population Sciences, 2000; chapter 7. Available at: http://www.nfhsindia.org/data/india/ indch7.pdf (Accessed on September 1, 2004)

Indian Journal of Pediatrics, Volume 71-November, 2004 


\section{Controlling Iron Deficiency Anemia Through the Use of Home-Fortified Complementary Foods}

8. UNICEF/Bangladesh Bureau of Statistics. Report on anaemia prevalence survey of urban Bangladesh and the rural Chittagong Hill Tracts 2003. May 2004.

9. Gibson RS, Ferguson EL, Lehrfeld J. Complementary foods for infant feeding in developing countries: their nutrient adequacy and improvement. Eur J Clin Nutr 1998; 52 : 764-770.

10. WHO. Complementary feeding of young children in developing countries: A review of current scientific knowledge. Geneva: World Health Organization, 1998.

11. Bhutta ZA. Iron and zinc intake from complementary foods: some issues from Pakistan. Pediatrics 2000; 106 : 1295-1297.

12. Dewey KG, Brown KH. Update on technical issues concerning complementary feeding of young children in developing countries and implications for intervention programs. Food Nutr Bull 2003; 24 : 5-28.

13. Horton S, Ross J. The economic of iron deficiency. J Food Policy 2003; $28: 51-75$.

14. Kapil US. Technical consultation on "Strategies for Prevention and Control of Iron Deficiency Anemia amongst under three children in India. Indian Pediatr 2002; 39 : 640-647.

15. Nestel P, Alnwick D. Iron/multi-micronutrient supplements for young children. Summary and conclusions of consultation held at UNICEF, Copenhagen, August 19-20, 1996. Washington, DC: International Life Sciences Institute, 1997.

16. INACG. Efficacy and effectiveness of interventions to control iron deficiency and iron deficiency anemia. International Nutritional Anemia Consultative Group. Washington, DC: International Life Sciences Institute, 2004.

17. Galloway R, McGuire J. Determinants of compliance with iron supplementation: supplies, side effects, or psychology? Soc Sci Med 1994; 39 : 381-390.

18. WHO. Iron deficiency anemia assessment, prevention and control: a guide to programme managers. Geneva: World Health Organization, 2001.

19. National guidelines on infant and young child feeding. Ministry of Human Resources Development, Department of Women and Child Development Food and Nutrition Board. Government of India, 2004. Department of Women and Child Development Food and Nutrition Board

20. WHO. Malnutrition. The global picture. Geneva: World Health Organization, 2000.

21. Zlotkin S, Arthur P, Antwi KY, Yeung G. Treatment of anemia with microencapsulated ferrous fumarate plus ascorbic acid supplied as sprinkles to complementary (weaning) foods. Am J Clin Nutr 2001; 74 : 791-795.

22. Zlotkin S, Antwi KY, Schauer C, Yeung G. Use of microencapsulated iron(II) fumarate sprinkles to prevent recurrence of anaemia in infants and young children at high risk. Bull World Health Organ 2003; 81 : 108-115.

23. Zlotkin S, Arthur P, Schauer C, Antwi KY, Yeung G, Piekarz A. Home-fortification with iron and zinc sprinkles or iron sprinkles alone successfully treats anemia in infants and young children. J Nutr 2003; 133 : 1075-1080.

24. Hyder SMZ, Zlotkin SH, Haseen F, Zeng L. Efficacy of daily versus weekly home fortification of weaning foods with Sprinkles among infants and young children in Bangladesh. Dhaka: National workshop on home fortification of weaning food with sprinkles: a new strategy to control iron deficiency anaemia among infants and young children, BRAC/IPHN/ MI/HSC, 2004

25. Tondeur MC, Schauer C, Christofides A, Asante KP, Newton $\mathrm{S}$, Serfass RE, Zlotkin SH. Determination of iron absorption from intrinsically labeled microencapsulated ferrous fumarate (sprinkles) in infants with different iron and hematologic status by using a dual-stable-isotope method. AJCN 2004 (In press).

26. Zlotkin S, Christofides A, Schauer C, Asante KP, Owusu-Agyei $\mathrm{S}$. Home fortification using sprinkles containing $12.5 \mathrm{mg}$ of iron successfully treats anemia in Ghanaian infants and young children. FASEB J 2004; 343.2.

27. Zlotkin S, Schauer C, Christofides A, Sharieff W, Hyder SMZ. Micronutrient Sprinkles to control childhood anemia: addressing an unresolved global problem through innovation, focused research and partnerships. PLOS 2004 (In press).

28. Christofides A, Zlotkin S, Schauer C. Impact of micronutrient sprinkles for the treatment and prevention of iron deficiency in Canadian first nations and Inuit infants 4-18 months old. FASEB J 2003; 17A; S1102.

29. Chan M, Zlotkin S, Yin SA, Sharieff W, Schauer C. The role of microencapsulated ferrous fumarate sprinkles in improving iron status and cognitive ability in preschool children in Northern China. Abstract, International Nutritional Anemia Consultative Group (2003) INACG Symposium. February 6, 2003, Marrakech Morocco, pp42. ILSI Research Foundation, Washington, DC. 


\section{Millennium Publications of Perennial Interest}

1. Pediatric Emergency Medicine ............................................. Rs 125

2. Pediatric Epilepsy (New Edition) …................................... Rs 125

3. Optimum Pulmonary Care of Neonates ........................... Rs 100

4. Pediatric Tuberculosis (New Edition) ................................ Rs 100

5. Vaccines for India in the New Millennium ........................ Rs 50

6. Cough in Children ............................................................ Rs 50

7. Rational Use of Antibiotics ............................................... Rs 50

8. Keeping the Muscles Strong in Muscular Dystrophy ....... Rs 50

\section{For Order Contact :}

Editor-in-Chief

The Indian Journal of Pediatrics

125 (2nd Floor), Gautam Nagar, New Delhi-110049

Post Box No. 3875, New Delhi - 110049.

Phone : 26568098. Telefax : 26857587

E-mail : ijp.journal.vsnl.net@vsnl.net

ijp@vsnl.net 